\title{
Validation Study of the Abbreviated Math Anxiety Scale: Spanish Adaptation
}

\author{
Jennifer L. Brown ${ }^{1, *} \&$ Lucía Macías Sifuentes ${ }^{2}$ \\ ${ }^{1}$ Department of Teacher Education, Columbus State University, Columbus, Georgia \\ ${ }^{2}$ Centro de Exito Estudiantil, Universidad de Monterrey, Monterrey, Mexico \\ *Correspondence: 4225 University Avenue, Columbus, Georgia 31907-5645. Tel: 706-569-3118. E-mail: \\ brown_jennifer2@columbusstate.edu
}

Received: October 3, $2016 \quad$ Accepted: October 24, $2016 \quad$ Online Published: November 17, 2016

doi:10.5430/jct.v5n2p76 URL: http://dx.doi.org/10.5430/jct.v5n2p76

\begin{abstract}
With growing numbers of Hispanic students enrolling in post-secondary school, there is a need to increase retention and graduation rates. The purpose of this study was to validate the Spanish adaptation of the Abbreviated Math Anxiety Scale (AMAS). The AMAS was translated and administered to 804 freshman students at a post-secondary institution in Mexico. After data analysis, the measure was found to be valid and reliable. The validated measure could serve as a predictor for math course grades and retention among Hispanic students at the post-secondary level, and it could allow researchers to study the structure of math anxiety across cultures.
\end{abstract}

Keywords: math anxiety; post-secondary; validation; Spanish

\section{Introduction}

\subsection{Introduce the Problem}

Spanish has the fastest projected growth among all of the world languages. Today, more than 500 million people speak the Spanish language. Furthermore, it is the second largest native population worldwide behind Chinese (Ministry of Foreign Affairs and Cooperation, 2014). According to Kena et al. (2015), 49.8 million students were enrolled in US public schools in 2013, and nearly a quarter of them classification themselves as Hispanic. This enrollment number has doubled from a decade ago. Despite these growing numbers, only one measure of math anxiety is available in the Spanish language.

\subsection{Review of Literature}

The concept of math anxiety began with Gough (1954). He coined the term, "mathemaphobia". A few years later, Dreger and Aiken (1957) defined number anxiety as "a syndrome of emotional reactions to arithmetic and mathematics" (p. 344). These researchers defined the concept, but it was nearly two decades before the construct was measured. Richardson and Suinn (1972) developed the Mathematics Anxiety Rating Scale (MARS), which contained 98 items. This measure was intended for secondary and post-secondary student populations. The MARS was later revised by Plake and Parker (1982) and shortened by Alexander and Martray (1989), which contained 25 items. The shortened MARS was translated into Spanish and validated by Núñez-Peña, Suárez-Pellicioni, Guilera, and Mercadé-Carranza (2013).

With the intent of developing a more parsimonious measure, Hopko, Mahadevan, Bare, and Hunt (2003) developed the Abbreviated Math Anxiety Scale (AMAS) based on the revised MARS (Plake \& Parker, 1982). The sample included 1,239 undergraduate students divided among three phases: developmental, primary, and replication. After the items were developed with the developmental sample, an exploratory factor analysis was conducted with the primary sample. Two factors (i.e., learning and assessment) emerged with strong factor loadings for each item, ranging from .52 to .89. The alpha coefficients for internal consistency ranged from .85 to .90 , and the alpha coefficients for test-retest reliability ranged from .78 to .85. This 9-item measure was composed of two subscales. One subscale contained five items that assessed learning math anxiety, and the other subscale contained four items that measured assessment math anxiety. The measure was found to have convergent and discriminant validity $(r=$ 
62). Using a confirmatory factor analysis with the replication sample, the researchers found the empirical data fit the theoretical structure. The mean math anxiety score was 23.2 with a standard deviation of 5.8.

Math anxiety can be experienced in a variety of settings ranging from the kitchen to the classroom (Ashcraft \& Moore, 2009). The causes of math anxiety could be environmental (e.g., a child's teacher who has a weakness in math) or could be related to one's information processing. Ashcraft and Kirk (2001) found the participants with high math anxiety had smaller working memory capacity $(M=2.8)$ compared to participants with low levels of math anxiety $(M=4.3)$, particularly with computational tasks. Using the AMAS with incoming college freshmen, Andrews and Brown (2015) found the relationship tended to be stronger between the students' final math course grades and the assessment subscale, including participants with an A course average. In addition to the relationship with math course grades, Betz (1978) connected math anxiety with college persistence. At least one math course will be required during the first 2 years of post-secondary education. Andrews and Brown (2015) found some participants delayed enrollment in a math course until the end of those 2 years.

During the fall semester of 2013, $15 \%$ of the undergraduate students at 4-year public institutions and $22 \%$ of the students at 2-year public institutions within the US indicated themselves as Hispanic (Kena et al., 2015). Hispanic students tend to earn an associate's degree instead of a bachelor's degree (Excelencia in Education, 2015). During 2010-2011, 41\% of Hispanic students graduated within 6 years with a bachelor's degree (Santiago \& Galdeano, 2014). In addition, 58\% of Hispanic students at 2-year institutions needed remediation at the post-secondary level (Excelencia in Education, 2015).

The majority of the research with math anxiety occurs in the US (Cipora, Szczgiel, Willmes, \& Nuerk, 2015). According to Ashcraft and Moore (2009), approximately 17\% of the population could exhibit high levels of math anxiety. Some cultures could have higher levels of math anxiety because of the high levels of importance placed on mathematical achievement and academic performance (Dowker, Sarkar, \& Looi, 2016). Lee (2009) found inconsistent math anxiety levels among high-achieving countries. Asian countries tended to have high levels of math anxiety, but Western European countries tended to have low levels of math anxiety. By having the same measure with various cultural adaptations, researchers could examine the structure of math anxiety across cultures and make comparisons (Cipora et al., 2015). A few researchers have validated the different cultural adaptations of the AMAS. Cipora, Szczygiel, Willmes, and Nuerk (2015), who validated the Polish adaptation of the AMAS, found the measure to have internal consistency (LMA $\alpha=.78$; MEA $\alpha=.84)$ and to have convergent and discriminant validity $(r=.49$ ). The mean score was 21.9 with a standard deviation of 6.6. Primi, Busdraghi, Tomasetto, Moranyi, and Chiesi (2014) validated the Italian adaptation of the AMAS. They found their measure to have internal consistency (LMA $\alpha=.80$; MEA $\alpha=.83)$ and to have convergent and discriminant validity $(r=.50)$. The mean score was 21.6 with a standard deviation of 6.3. Vahedi and Farrokhi (2011) validated the Persian adaptation of the AMAS. They found their measure to have internal consistency (LMA $\alpha=.75$; MEA $\alpha=.79$ ) and to have convergent and discriminant validity $(r=.50)$. The mean score was 18.4 with a standard deviation of 6.8 .

\subsection{Purpose of the Study}

The purpose of this study was to validate the Spanish adaptation of the Abbreviated Math Anxiety Scale (AMAS). Thus, the final product could offer another option for assessing math anxiety of secondary and post-secondary students in the Spanish language. In addition, the measure could be used to examine math anxiety cross-culturally.

\section{Method}

\subsection{Participants}

The setting for this study was a large university in Mexico with a student population of 12,000 who ranged from high school to postgraduates. The 1,600 freshman students were invited to participate, and 1,102 students responded, which was a $69 \%$ response rate. Of those 1,102 cases, 804 were deemed valid. These 804 cases were split randomly into two groups for the exploratory and confirmatory factor analyses. The exploratory factor analysis (EFA) group contained 404 participants, including $133(32.9 \%)$ males and $271(67.1 \%)$ females. The confirmatory factor analysis (CFA) group contained 400 students, including 139 (34.75\%) males and 261 (65.25\%) females.

\subsection{Data Collection}

\subsubsection{Procedures}

The invitation to participate was sent via institutional email. Within the email, an anonymous link for the web-based survey was included. The participants could select the link or copy and paste it into their internet browser. The first 
page of the web-based survey included the purpose of the study and other items from the informed consent. The participants selected the appropriate radial within the web-based survey as to whether they agreed or disagreed to participate in the study. If they chose not to participate, the survey concluded, and the response was recorded. If they chose to participate, then they responded to each of the survey items. The AMAS (Hopko et al., 2003) was administered along with the Freshman Orientation Survey (Brown, 2012). The total amount of time needed to complete the measures was 20 minutes.

\subsubsection{Measure}

The AMAS (Hopko et al., 2003) contained nine items, which were divided into two subscales, Learning Math Anxiety (LMA) and Math Evaluation Anxiety (MEA). LMA contained five items that measures math anxiety during math instructional or studying time. MEA contained four items that measures math anxiety during administration of math assessments. The response scale ranged from 1, which represented Low, to 5, which represented High. Hopko and his colleagues validated the measure with 1,239 undergraduate students using exploratory factor analysis and confirmatory factor analysis and found the measure had good internal consistency (LMA $\alpha=.85$ and MEA $\alpha=.88$ ), 2-week test-retest reliability (LMA $\alpha=.78$ and MEA $\alpha=.83$ ), and good convergent validity (LMA and total AMAS score $r=.88$ and MEA and total AMAS score $r=.92)$ and divergent validity $(r=.62)$. The measure was translated into Spanish by the researchers. Then, the measure was administered as an addendum to the Freshman Orientation Survey (Brown, 2012).

\section{Results}

\subsection{Exploratory Factor Analysis}

To validate the measure in its Spanish adaptation, an exploratory factor analysis using principal axis factoring with an oblimin rotation was conducted with the EFA group. The purpose of this factor analysis was to discover the factor structure of the selected items and the correlations between the factors. This method consisted of a principal component analysis followed by rotation of factors that had eignvalues greater than 1.00. A criterion of .40 or higher was established for the structure coefficients (Meyers, Gamst, \& Guarino, 2006). The Kaiser-Meyer-Olkin (KMO) Measure of Sampling is a summary index, which ranges from 0 to 1 , of the interrelatedness among the items and the extent to which the items will yield an appropriate factor analysis. With the randomly selected sample, the KMO was .90 , which exceeds the suggested minimum value of .70 . Bartlett's Test of Sphericity (approximate $\chi^{2}=5210.87$, $p<.001)$ indicates that the intercorrelations among the items are statistically significant and of a sufficient magnitude to conduct a factor analysis (Meyers et al., 2006). Using the nine items, two factors emerged with initial eigenvalues above 1.0. The communalities ranged from .42 to .82 . With this analysis, $70.45 \%$ of the variance was explained. Tables 1 and 2 present the specific items with their respective factor loadings for each subscale. Reliability analyses were conducted for the two factors to determine internal consistency among the scale items. The alpha coefficient for Learning Math Anxiety was .883, and the alpha coefficient for Math Evaluation Anxiety was .867. The alpha coefficient for all items was .917. Using the Cohen (1988) guidelines, the results indicated that the coefficients were good, and the items were internally consistent. The mean math anxiety score for the EFA group was 25.37 with a standard deviation of 9.10 .

Table 1. Factor 1 (Learning Math Anxiety)

\begin{tabular}{lc}
\hline \multicolumn{1}{c}{ Item } & Loading \\
\hline Having to use the tables in the back of a math book. & .569 \\
Watching a teacher work an algebraic equation on the blackboard. & .811 \\
Listening to a lecture in math class. & .954 \\
Listening to another student explain a math formula. & .894 \\
Starting a new chapter in a math book. & .818 \\
\hline
\end{tabular}


Table 2. Factor 2 (Math Evaluation Anxiety)

\begin{tabular}{lc}
\hline \multicolumn{1}{c}{ Item } & Loading \\
\hline Thinking about an upcoming math test one day before. & .733 \\
Taking an examination in a math course. & .711 \\
Being given a homework assignment of many difficult problems which is due the next & .875 \\
class meeting. & .915 \\
Being given a "pop" quiz in a math class.
\end{tabular}

\subsection{Confirmatory Factor Analysis}

Using the CFA group, a confirmatory factor analysis was conducted using AMOS 21.0 to determine how the theoretical structure fits with the data and to cross-validate the factor structures created with the exploratory factor analysis. According to Meyers et al. (2006), acceptable model fit indexes include the Comparative Fit Index (CFI) and the Root Mean Square Error of Approximation (RMSEA). A value of .95 or greater for the CFI is deemed as an acceptable fit. For the RMSEA, a value of .08 or less indicates good fit. The model, presented in Figure 1, had a significant chi-square $\left(\chi^{2}=181.024 ; d f=26 ; p<.00\right)$ and a low CFI (.936) and high RMSEA (.113). The initial hypothesized model was rejected based on the goodness-of-fitness statistics. Using the modification indices, a restricted model, presented in Figure 2, was created. The model had a higher CFI (.974), and the RMSEA value was .074, which improved by .039 . The chi-square was significant $\left(\chi^{2}=86.335 ; d f=24 ; p<.001\right)$. All of these indices indicated a good fit between the restricted model and the empirical data. Based on these results, the model was deemed to have good fit with the data. Table 3 displays the standardized beta weights for each item.

To determine the internal consistency among the scale items with the CFA sample, another set of reliabilities analyses were conducted. The alpha coefficient for Learning Math Anxiety was .883, and the alpha coefficient for Math Evaluation Anxiety was .855. The alpha coefficient for all items was .901. The results indicated that the coefficients were good based on Cohen's guidelines (1988), meaning the items were internally consistent, and they were similar to the EFA group's alpha coefficients. The mean math anxiety score for the CFA group was 25.13 with a standard deviation of 8.90. To measure discriminant validity, a bivariate correlation was conducted using the two scales, Learning Math Anxiety and Math Evaluation Anxiety $(r=.724)$. With a correlational coefficient less than or equal to .80 as a criterion (Meyers et al., 2006), these results suggested that the factors had discriminant validity and were not measuring the same concept.

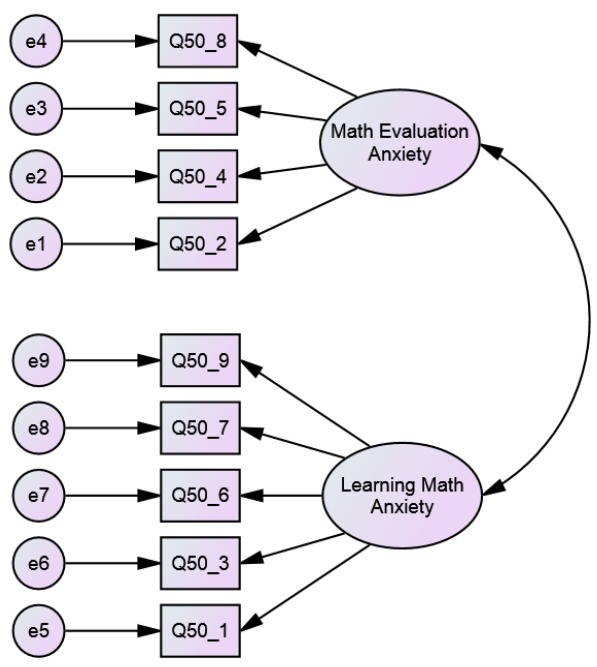

Figure 1. Original Model 


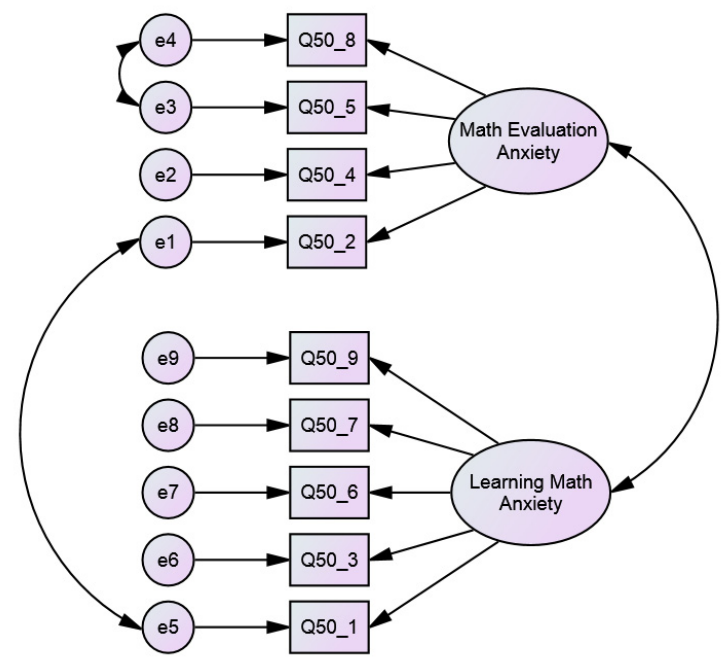

Figure 2. Restricted Model

Table 3. Standardized Beta Weights by Confirmatory Factor Analysis

\begin{tabular}{ccc}
\hline & \multicolumn{2}{c}{ Factor } \\
\cline { 2 - 3 } Item & Learning Math Anxiety & Math Evaluation Anxiety \\
\hline Q50_1 & .529 & \\
Q50_3 & .842 & \\
Q50_6 & .858 & \\
Q50_7 & .798 & \\
Q50_8 & .800 & .630 \\
Q50_2 & & .964 \\
Q50_4 & & .670 \\
Q50_5 & & .684 \\
Q50_8 & & \\
\hline
\end{tabular}

\section{Discussion}

Based on the cross-validation data analysis using an exploratory factor analysis and confirmatory factor analysis, the Spanish adaptation of the AMAS was found to have convergent and discriminant validity in addition to internal consistency among the items. For the Spanish adaptation, the mean math anxiety score was higher (i.e., 25) compared to the other adaptations, but it was similar to the replication sample (i.e., 23) from Hopko et al. (2003). The factor loadings were consistent with the original validation study by Hopko et al. (2003), ranging from .529 to .964 . The alpha coefficients for internal consistency were similar to the other validation studies (i.e., Polish, Italian, and Persian), which ranged from .75 to .85 . The correlational coefficient used to determine discriminant validity was higher with the Spanish adaptation $(r=.724)$ compared to the four other measures, but the established criterion was met.

Based on special education litigation and legislation, assessment in native language is a requirement of current special education law (i.e., Individuals with Disabilities Education Act of 2004). Assessment in students' native language is a better option if valid measures are available. Depending on the students' language abilities, they may read the prompt in English, translate it into their native language, respond to the prompt in their native language, and translate their response back into English. This long and difficult task affects the length of time to complete the assessment and could increase their anxiety levels (Figueroa, 1989). This validated measure could assist with reducing that anxiety. In addition, it could serve as a predictor of math course grade and retention of Hispanic 
students at the post-secondary level. Lastly, with four validated adaptations of the AMAS, researchers will be able to examine the structures of math anxiety across five different cultures.

\section{References}

Alexander, L., \& Martray, C. (1989). The development of an abbreviated version of the mathematics anxiety rating scale. Measurement and Evaluation in Counseling and Development, 22, 143-150.

Andrews, A., \& Brown, J. L. (2015). The effects of math anxiety. Education, 135(3), 362-370.

Ashcraft, M. H., \& Kirk, E. P. (2001). The relationships among working memory, math anxiety, and performance. Journal of Experimental Psychology: General, 130(2), 224-237. http://dx.doi.org/10.1037//0096-3445.130.2.224

Ashcraft, M. H., \& Moore, A. M. (2009). Mathematics anxiety and the affective drop in performance. Journal of Psycheducational Assessment, 27(3), 197-205. http://dx.doi.org/10.1177/0734282908330580

Betz, N. E. (1978). Prevalence, distribution and correlates of math anxiety in college students. Journal of Counseling Psychology, 25(5), 441-448. http://dx.doi.org/10.1037/0022-0167.25.5.441

Brown, J. L. (2012). Development of a freshman orientation survey to improve student retention within a college. College Student Journal, 46(4), 834-851.

Cipora, K., Szczygiel, M., Willmes, K., \& Nuerk, H. -C. (2015). Math anxiety assessment with the abbreviated math anxiety scale: Applicability and usefulness: Insights from the Polish adaptation. Frontiers in Psychology, 6, 1-16. http://dx.doi.org/10.3389/fpsyg.2015.01833

Cohen, J. (1988). Statistical power analysis for the behavioral sciences (2nd ed.). Hillsdale, NJ: Erlbaum.

Dowker, A., Sarkar, A., \& Looi, C. Y. (2016). Mathematics anxiety: What have we learned in 60 years? Frontiers in Psychology, 7, 1-16. http://dx.doi.org/10.3389/fpsyg.2016.00508

Dreger, R. M., \& Aiken, L. R. (1957). The identification of number anxiety in a college population. Journal of Educational Psychology, 48, 344-351. http://dx.doi.org/10.1037/h0045894

Excelencia in Education. (2015). The condition of Latinos in education: 2015 factbook. Washington, D.C.: Excelencia in Education. Retrieved from http://www.nccpsafety.org/assets/files/library/The_Condition_of_Latinos_in_Education.pdf

Figueroa, R. A. (1989). Psychological testing of linguistic-minority students: Knowledge gaps and regulations. Exceptional Children, 56(2), 145-152.

Gough, M. F. (1954). Mathemaphobia: Causes and treatments. Clearing House, 28, 290-294.

Hopko, D. R., Mahadevan, R., Bare, R. L., \& Hunt, M. K. (2003). The abbreviated math anxiety scale (AMAS): Construction, validity, and reliability. Assessment, 10(2), 178-182.

Kena, G., Musu-Gillette, L., Robinson, J., Wang, X., Rathbun, A., Zhang, J., Wilkinson-Flicker, S., Barmer, A., \& Dunlop Velez, E. (2015). The condition of education 2015 (NCES 2015-144). Washington, DC: U.S. Department of Education, National Center for Education Statistics. Retrieved from http://nces.ed.gov/pubs2015/2015144.pdf

Lee, J. (2009). Universals and specifics of math self-concept, math self-efficacy, and math anxiety across 41 PISA 2003 participating countries. Learning and Individual Differences, 19, 355-365. http://dx.doi.org/10.1016/j.lindif.2008.10.009

Meyers, L. S., Gamst, G. C., \& Guarino, A. J. (2006). Applied multivariate research: Design and interpretation. Thousand Oaks, CA: Sage Publications.

Ministry of Foreign Affairs and Cooperation. (2014, Febrero). Spanish: An expanding universal language (501-14-005-4). Madrid, Spain: Ministerio de Asuntos Axteriores y de Cooperación, Gobierno de España. Retrieved from http://www.exteriores.gob.es/Portal/es/SalaDePrensa/Multimedia/Publicaciones/Documents/2014_IDIOMA_E N.pdf

Plake, B. S., \& Parker, C. S. (1982). The development and validation of a revised version of the mathematics anxiety rating scale. Educational and Psychological Measurement, 42(2), 551-557. http://dx.doi.org/10.1177/001316448204200218 
Primi, C., Busdraghi, C., Tomasetto, C., Morsanyi, K., \& Chiesi, F. (2014). Measuring math anxiety in Italian college and high school students: Validity, reliability and gender invariance of the abbreviated math anxiety scale (AMAS). Learning and Individual Differences, 34, 51-56.

Núñez-Peña, M. I., Suárez-Pellicioni, M., Guilera, G., \& Mercadé-Carranza, C. (2013). A Spanish version of the short mathematics anxiety rating scale (sMARS). Learning and Individual Differences, 24, 204-210.

Richardson, F. C., \& Suinn, R. M. (1972). The mathematics anxiety rating scale: Psychometric data. Journal of Counseling Psychology, 19(6), 551-554.

Santiago, D. A., \& Galdeano, E. C. (2014, April). Latino college completion: United States. Washington, DC: Excelencia in Education. Retrieved from http://www.edexcelencia.org/research/college-completion/united-states

Vahedi, S., \& Farrokhi, F. (2011). A confirmatory factor analysis of the structure of abbreviated math anxiety scale. Iranian Journal of Psychiatry, 6(2), 47-53.

\section{Notes}

Note 1. AMAS translated items

Tener que utilizar las tablas que están reverso del libro de matemáticas.

Un día antes, estar pensando sobre el próximo examen de matemáticas

Observar al profesor trabajar en una ecuación algebraica en el pizarrón

Tener un examen en una clase de matemáticas

Se me asigne una tarea con muchos problemas difíciles, para entregar en la siguiente clase.

Escuchar la clase de matemáticas.

Escuchar a otro estudiante explicar una fórmula matemática.

Tener un "examen sorpresa" en una clase de matemáticas

Iniciar un capítulo nuevo del libro de matemáticas 